\title{
An Active Guarding Technique for Substrate Noise suppression on LC-tank Oscillators
}

\author{
Hao-Ming Chao ${ }^{(1,2)}$, Kuei-Ann Wen ${ }^{(1)}$ \\ ${ }^{1}$ Department of Electronics Engineering \\ \& Institute of Electronics, National Chiao Tung University \\ 1001 Ta Hsueh Road, Hsinchu 300, Taiwan
}

\begin{abstract}
An active guarding technique is demonstrated for substrate immunity improvement on LC-tank oscillators. Active components in the oscillator, which are sensitive to substrate noise and contribute to the output most, are guarded from the substrate perturbation by applying the active guarding circuit to decouple and also compensate the noise current. To evaluate the effectiveness of the proposed technique, noise signals of both single and multiple tones of various frequencies are injected to the substrate close to the guarded transistors and measure the sideband spurs by switching the active guarding circuit on/off respectively. Experimental results showed more than $11 \mathrm{~dB}$ noise suppression performances can be achieved in a wide frequency range from DC to $10 \mathrm{GHz}$. By applying the active guarding circuit to a LC-tank oscillator, more than $17 \mathrm{~dB}$ spur suppression performance is obtained in the frequency range of interest.
\end{abstract}

\section{INTRODUCTION}

During the past years, a trend of higher level integration as system-on-chip (SoC) design has grown enormously, driven by the advancement of the continuous down-scaling CMOS technologies. However, this trend leads to a serious problem of signal integrity degradation due to the impact of crosstalk through the shared substrate. This problem has drawn more attention recently thus researches of various respects have been investigated [1]-[6]. In this work, we focus on the substrate noise suppression on LC-tank oscillator because of its high sensitivity to noises and also the important role of generating the reference frequency for phase-locked loop (PLL), which makes it one of the most crucial building blocks among a wireless communication system.

A similar active substrate noise suppression experiment on ring oscillator has been presented in [5], which uses an inverting amplifier to perform the noise cancellation and capacitively sense/transmit the noise signal to substrate through a pair of concentric guard rings. However, as reported in the paper, at low frequencies, the noise cancellation ability is limited by the capacitive coupling between the guard rings and the amplifier; at high frequencies, the performance is also limited by the bandwidth of the amplifier, even if SiGe technology is already being used. Therefore, the bandwidth for $10 \mathrm{~dB}$ noise suppression performance is only from around $5 \mathrm{MHz}$ to $200 \mathrm{MHz}$.

In this work, we demonstrate an active guarding technique for substrate noise suppression and apply it to a LC-tank oscillator to alleviate the impact of substrate noise. Active components in the oscillator are protected by an active guarding circuit. Noise decoupling and feed-forward

\author{
Michiel Steyaert ${ }^{(2)}$ \\ ${ }^{2}$ Katholieke Universiteit Leuven \\ Department of Electrical Engineering, MICAS \\ 3001 Heverlee, Belgium
}

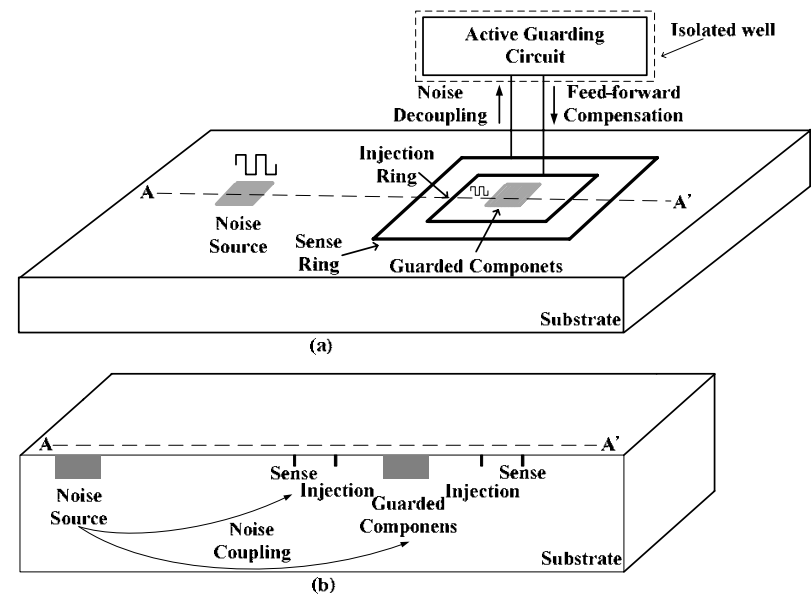

Fig. 1 (a) Active guarding technique (b) Cross-section of the physical implementation

compensation mechanisms in current mode are developed in the technique to avoid the using of power consuming and bandwidth limited amplifiers like in [4]-[6], thus achieves a wideband characteristic of more than $11 \mathrm{~dB}$ noise suppression performance from DC to $10 \mathrm{GHz}$. In the end, DCS-1800 spurious suppression specifications are taken as an example to reveal a possible utilization of the proposed technique.

This paper is organized as follows. Section II describes the active guarding technique for substrate noise suppression and the application to a LC-tank oscillator. Section III shows the experimental results. Discussions and conclusions will be given in Section IV.

\section{ACTIVE GUARDing TECHNIQUE ON LC-TANK OSCILLATOR}

The first version of active guarding technique was proposed by the authors in [3], and is now being optimized for high frequency operation of more than $10 \mathrm{GHz}$ bandwidth with 11 $\mathrm{dB}$ noise suppression performance.

\section{A. Active Guarding Technique}

As shown in Fig. 1, an active guarding circuit with a pair of concentric rings (sense and injection) is used to suppress the substrate coupling noise between the noise source and guarded components. The sense ring is placed at the outer side to receive the substrate noise; the injection ring, on the contrary, surrounds the guarded components at the inner side to inject the compensation current back to the substrate. In order to 


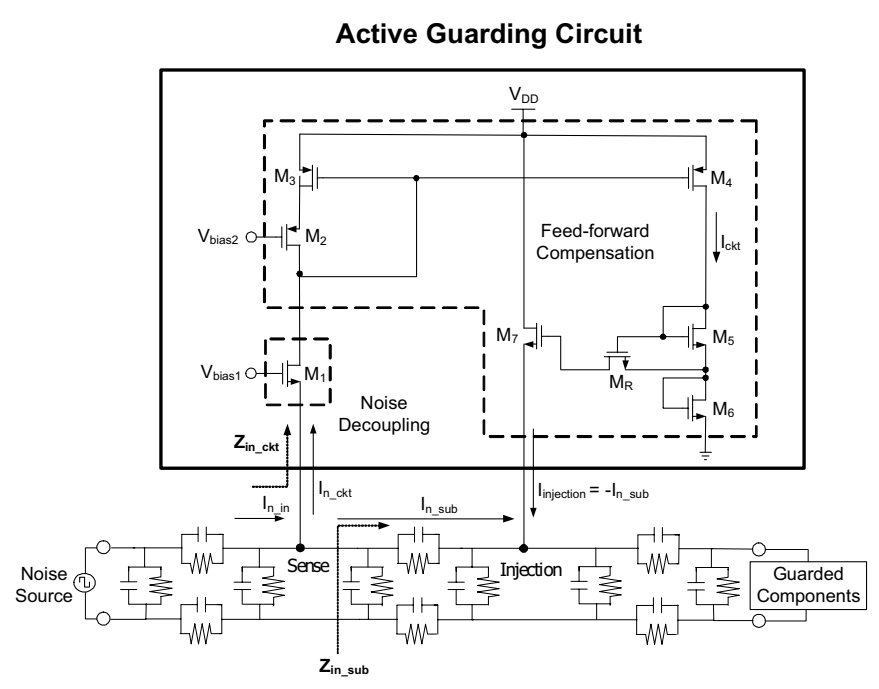

Fig. 2. Active guarding circuit

prevent self-induced noise from interfering the guarded circuitry through the common substrate, the active guarding circuit is placed in an isolated well.

Fig. 2 shows the active guarding circuit, which performs two mechanisms as noise decoupling by transistor $M_{1}$ and feed-forward compensation by transistors of $M_{2}$ to $M_{7}$ and $M_{R}$. Noise current from any direction to the guarded components is detected and averaged at the sense ring as $I_{n-i n}$, and then decoupled to the active guarding circuit in a portion as $I_{n_{-} c k t}$ by the following equation:

$$
\frac{I_{n_{-} c k t}}{I_{n_{-} i n}}=\left(\frac{Z_{i n \_s u b}}{Z_{\text {in_ckt }}+Z_{\text {in_sub }}}\right)
$$

where $Z_{\text {in_sub }}$ is the substrate impedance seen from the sense node and $Z_{\text {in ckt }}$ is the input impedance of a common gate configured transistor $M_{1}$, which equals to $1 /\left(g_{m l}+\mathrm{S} C_{g s l}\right)$.

As a consequence, the noise current will be partially drained to the active guarding circuit through the low impedance path of $1 / g_{m l}$, and then become the source for generating the noise cancellation current in the later stage. As to the noises with frequency higher than the bandwidth of the active guarding circuit, they will be decoupled away through $C_{g s 1}$.

The feed-forward compensation mechanism includes the operations of -180 degree phase inversion, amplitude adjustment and bandwidth extension. The -180 degree phase inversion of the decoupled noise current $I_{n c k t}$ is accomplished by a current mirror composed of $M_{2}$ to $M_{4}$. The phase-inversed current $I_{c \text { ckt }}$ is sent to the amplitude controller composed of $M_{5}$ to $M_{7}$ and $M_{R}$ with current gain $A_{C}(s)$ to adjust the magnitude of the compensation current $I_{\text {injection }}$ to be identical to $I_{n \text { sub }}$, as shown in equation (2) and (3). However, the remaining noise current $I_{n_{\text {s sub }}}$ can flow through both the surface and the bottom portions of the substrate network. By designing the injection ring to be placed closely adjacent to the sense ring, the surface portion of substrate network in between is a low impedance path and dominates the current

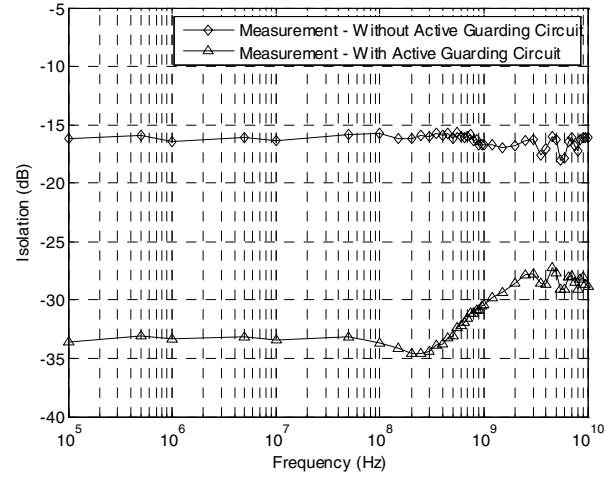

Fig. 3 Measurement results of the isolation improvement by the protection of the active guarding circuit

flow of $I_{n_{-} s u b}$. As a result, the magnitude-matched and phasereversed current $I_{\text {injection }}$ is injected to the substrate through the injection ring and mixed with $I_{n \leq s u b}$ for the cancellation of the total noise received at the guarded components.

$$
\begin{gathered}
I_{\text {Injection }}=I_{n_{-} c k t} \times A_{C}(s)=-I_{n_{-} s u b} \\
\left|A_{C}(s)\right|=\frac{I_{n_{-} s u b}}{I_{n_{-} c k t}}=\frac{Z_{\text {in_ckt }}}{Z_{\text {in_sub }}}
\end{gathered}
$$

To release the bandwidth limitation of the current mirror which performs the phase inversion, frequency compensation is performed by introducing a zero with the adding of transistors $M_{5}, M_{6}$ and $M_{R}$. Other details about active guarding circuit design with the demonstration of a first prototype can be found in [3].

\section{B. LC-tank Oscillator}

A conventional complementary negative transconductance LC-tank oscillator is used for simplicity in this work, as shown in Fig. 4. Substrate noise can couple into the oscillator under test through many ways, as from the $\mathrm{p}+$ contacts that connect the substrate to the on-chip ground, the active devices' back gates and also the components made of metal layers like inductors and capacitors. The dominant mechanism that converts substrate noise to the output of the LC-tank oscillator will be AM modulation of the bias transistor current due to the noise coupled to its ground. Both the AM sidebands from low frequency substrate noise harmonics and the impact of indirect AM to FM conversion due to the substrate noise on bias transistor are described in the following equation from [2],:

$$
\begin{aligned}
L\left\{\omega_{m}\right\}= & S_{\text {sub }}\left(\omega_{0} \pm \omega_{m}\right)\left(\frac{g_{m}^{\text {bias }}}{12 \sqrt{2} I_{\text {bias }}}\right)^{2}\left(\frac{\omega_{0}}{2 Q \omega_{m}}\right)^{2} \\
& +S_{\text {sub }}\left(\omega_{m}\right)\left(\frac{d \omega_{m}}{d I_{\text {bias }}}\right)^{2}\left(g_{m}^{\text {bias }}\right)^{2}\left(\frac{1}{2 \omega_{m}^{2}}\right)
\end{aligned}
$$

where $S_{\text {sub }}(\omega)$ is the substrate noise signal at, $\omega_{0}$ is the resonant frequency, $Q$ is the quality factor of the tank, $I_{\text {bias }}$ and $g_{m}^{\text {bias }}$ are the current and transconductance of bias transistor, respectively. 


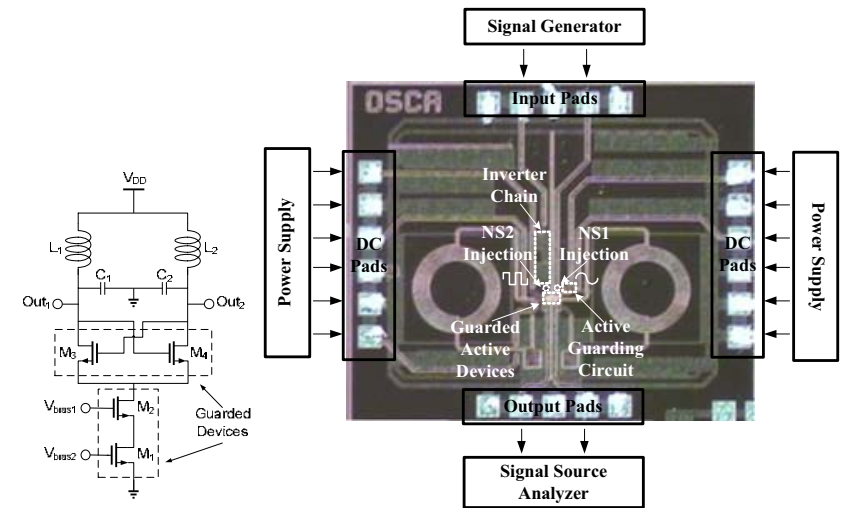

Fig. 4 The microphotograph of the test chip, schematic of the LC-tank oscillator and the experimental setup

Consequently, to minimize the substrate noise impact on the bias transistor that modulates the output of the oscillator, the active substrate noise suppression technique is applied to guard the bias transistors $M_{1}$ and $M_{2}$, thus the parameters of $I_{\text {bias }}$ and $g_{m}^{\text {bias }}$ (also $g_{m b}^{\text {bias }}$ ) of both transistors will be more stable to substrate noise. In addition, transistors $M_{3}$ and $M_{4}$ which composes the cross-coupled pair are also being protected, since they suffer from the substrate noise through their back gate that modulates their threshold voltages, too.

\section{EXPERIMENTAL RESULTS}

Two $0.18 \mu \mathrm{m}$ CMOS chips were fabricated for a complete experiment. The first one simply contains an active guarding circuit to evaluate the performance itself. The second chip integrates an active guarding circuit and a LC-tank oscillator to verify the applicability for real applications. Sideband spurs and phase noise performance are measured to evaluate the effectiveness of the proposed technique. The microphotograph of the integrated chip, the schematic of the LC-tank oscillator and the experimental setup are shown in Fig. 4.

\section{A. Active Guarding Circuit}

The active guarding circuit used in the integrated chip is fabricated in a separate chip for performance verification. The performance shown in Fig. 3 is obtained by measuring the isolation between two $10 \mu \mathrm{m} \times 10 \mu \mathrm{m} \mathrm{p}+$ diffusion pick-ups with $30 \mu \mathrm{m}$ distance with/without the protection of the active guarding circuit, respectively. Instead of using printed circuit board with bonding wires for measurement in our previous design [4], this work uses on wafer measurement to release the bond wire and loading effects that degrade the high frequency performance. More than $14 \mathrm{~dB}$ noise suppression performance is achieved in a wide frequency range from $\mathrm{DC}$ to $1 \mathrm{GHz}$ and $11 \mathrm{~dB}$ until $10 \mathrm{GHz}$, which is sixty-six times wider than our previous work in [3], by the cost of a small chip area of $20 \mu \mathrm{m} \times 40 \mu \mathrm{m}$ and $2 \mathrm{~mA}$ current consumption from a $1.8 \mathrm{~V}$ supply. Table I summarize the performance of this work with comparison to other recently published active substrate noise suppression circuits with measurement results. This work achieves widest noise suppression bandwidth with smallest area consumption while using the oldest technology.
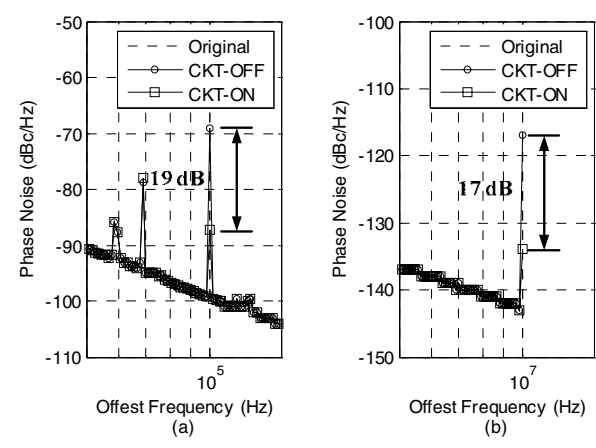

Fig.5. Comparison of the phase noise differences among the Original/CKT$O N / C K T-O F F$ after noise injections of (a) $100 \mathrm{KHz}$ (b) $10 \mathrm{MHz}$ from NS1.

\section{B. Integrated chip}

As shown in Fig. 4, an active guarding circuit and a LCtank oscillator are integrated in a single chip to evaluate the effectiveness of the proposed technique on real applications. The LC-tank oscillator is verified with measurement results to operate at $1 \mathrm{GHz}$ and offers a phase noise performance of more than $-95 \mathrm{dBc} / \mathrm{Hz}$ at $100 \mathrm{kHz}$ offset frequency. It consumes $1.1 \mathrm{~mA}$ from a $1.8 \mathrm{~V}$ supply.

Two different kinds of noise sources are applied for the experiment. Noise source 1 (NSI) injects single tone signals from an external source as signal generator into the substrate through substrate taps, with $15 \mu \mathrm{m}$ distance from the guarded devices. Noise source 2 (NS2) is the digital signal output from an on-chip inverter chain, which is commonly used as clock buffers, for the emulation of the substrate noise source in practical cases. The output of the inverter chain is fed to the substrate with $25 \mu \mathrm{m}$ distance from the guarded devices, through a $5 \mathrm{pF}$ capacitor to pass signals below several gigahertz and also to block the DC signal from interfering the substrate. Noise frequencies of either low as $100 \mathrm{KHz}$ to 10 $\mathrm{MHz}$ or high as around the resonant frequency of $1 \mathrm{GHz}$ are injected to the substrate to see the impact of substrate coupling and the suppression ability of the active guarding circuit.

For each noise frequency, the phase noise or sideband spurs are measured under three different conditions as follows:

- Original: The active guarding circuit is off and no noise is injected.

- CKT-OFF: The active guarding circuit is off and noise is injected either from NS1 or NS2.

- CKT-ON: The active guarding circuit is on and noise is injected either from NS1 or NS2.

The effectiveness of the active guarding technique is obtained by comparing the performance differences between $C K T-O F F$ and $C K T-O N$ for noise injections of various frequencies. For low frequency range of interested, experimental results of $100 \mathrm{KHz}$ and $10 \mathrm{MHz}$ single tone noise injections from $N S 1$ with $-5 \mathrm{dBm}$ input power are presented in Fig. 5(a) and (b) as examples. As shown in the figure, the injected noise signals result in corresponding spurs at $100 \mathrm{KHz}$ and $10 \mathrm{MHz}$ offset frequency with spurs' power of $-117 \mathrm{dBc} / \mathrm{Hz}$ and $-69 \mathrm{dBc} / \mathrm{Hz}$, respectively. After the active guarding circuit is switched on, the spurs' power are suppressed to $-134 \mathrm{dBc} / \mathrm{Hz}$ and $-88 \mathrm{dBc} / \mathrm{Hz}$, which shows 
TABLE I

PERFORMANCE SUMMARY

\begin{tabular}{|c|c|c|c|c|c|}
\hline \multirow{2}{*}{ References } & \multirow{2}{*}{ Technology } & \multicolumn{2}{|c|}{ Noise Suppression Bandwidth } & \multirow{2}{*}{ Power } & \multirow{2}{*}{ Area } \\
\hline & & $11 \mathrm{~dB}$ Suppression & $3 \mathrm{~dB}$ Suppression & & \\
\hline This Work & $\begin{array}{l}180 \mathrm{~nm} \\
(\mathrm{CMOS})\end{array}$ & $\mathrm{DC}-10 \mathrm{GHz}$ & $>10 \mathrm{GHz}$ & $3.6 \mathrm{~mW}$ & $20 \mu \mathrm{m} \times 40 \mu \mathrm{m}$ \\
\hline T-CAD'09 [6] & $\begin{array}{c}180 \mathrm{~nm} \\
(\mathrm{BiCMOS})\end{array}$ & N/A & $200 \mathrm{MHz}-600 \mathrm{MHz}$ & $2 \mathrm{~mW}$ & $\stackrel{\text { N/A }}{(14 \mathrm{pF} \text { Capacitor + Circuit Area) }}$ \\
\hline T-MTT'08 [3] & $\begin{array}{l}90 \mathrm{~nm} \\
(\mathrm{CMOS})\end{array}$ & $\mathrm{DC}-150 \mathrm{MHz}$ & $\mathrm{DC}-650 \mathrm{MHz}$ & $2.5 \mathrm{~mW}$ & $20 \mu \mathrm{m} \times 41 \mu \mathrm{m}$ \\
\hline $\mathrm{JSSC}^{\prime} 05[4]$ & $\begin{array}{l}130 \mathrm{~nm} \\
(\mathrm{CMOS})\end{array}$ & $\mathrm{N} / \mathrm{A}$ & $170 \mathrm{MHz}-300 \mathrm{MHz}$ & $9.9 \mathrm{~mW}$ & $\frac{\text { N/A }}{(30 \mathrm{pF} \text { Capacitor + Circuit Area) }}$ \\
\hline
\end{tabular}

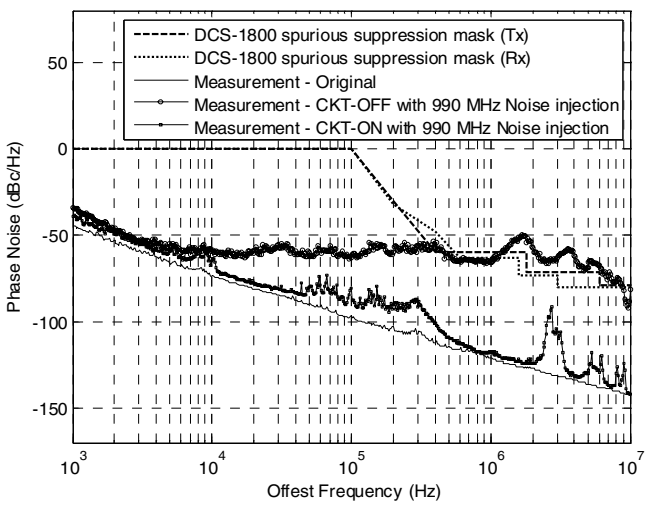

Fig. 6 Comparison of the phase noise differences among the Original/CKT$O N / C K T-O F F$ after $990 \mathrm{MHz}$ noise injection from NS2 with DCS-1800 spurious suppression masks.

more than $17 \mathrm{~dB}$ improvement for both injections. Two spurs in $60 \mathrm{KHz}$ and $70 \mathrm{KHz}$ in Fig. 5(a) are not resulting from substrate noise therefore cannot be suppressed. Similar results are obtained for noise frequencies from $100 \mathrm{KHz}$ to $10 \mathrm{MHz}$.

For high frequency experiments, here we show a result of the noise injection from NS2 as an example since the phase noise degradations due to the injections of digital noise are more severe than single tone noise for the same frequencies. A $990 \mathrm{MHz}$ high frequency noise, which is close to the resonant frequency of the oscillator, is injected to the substrate from NS2 as a digital noise to see the phase noise performance difference. Noise amplitude which reaches the guarded devices in the oscillator is calculated to be around $30 \mathrm{mV}$ to emulate the actual possible substrate noise.

Wireless standard of DCS-1800 is chosen as an example application for the oscillator design thus both transmission (Tx) and reception $(\mathrm{Rx})$ spurious suppression specifications calculated in [7] are being plotted in Fig. 6 for comparison. As shown in the figure, the oscillator output is suffered from $\mathrm{AM} / \mathrm{FM}$ modulations and the puling effect by the injected noise that the phase noise performance are degraded seriously therefore failed to fulfil the mask specifications of both $\mathrm{Rx}$ and $\mathrm{Tx}$, from offset frequencies of $500 \mathrm{kHz}$ and $1.1 \mathrm{MHz}$, respectively. After the active guarding circuit is switched on, the pulling effect is reduced hence the phase noise performances are improved substantially to pass both Tx and $\mathrm{Rx}$ spurious suppression masks in the whole frequency range of interest.

\section{CONCLUSIONS}

This paper demonstrates an active guarding technique to improve the substrate noise immunity on analog/RF circuits. Experimental results showed this work achieves widest bandwidth with $11 \mathrm{~dB}$ noise suppression bandwidth from DC to $10 \mathrm{GHz}$ with smallest area consumption while using the oldest technology while using the oldest technology, as summarized in Table I.

By applying the active guarding circuit to a LC-tank oscillator, more than $17 \mathrm{~dB}$ spur suppression performance is obtained in the frequency range of interest as either low frequencies below $10 \mathrm{MHz}$ or high frequencies around the resonant frequency of $1 \mathrm{GHz}$. As a result, the $1 \mathrm{GHz}$ oscillator can fulfil the spurious suppression masks of DCS-1800 even in the adjacent of $990 \mathrm{MHz}$ signals. Moreover, the active guarding circuit benefits better performance and lower power consumption as the technology scaling down; thus is suitable for future applications of highly integrated SoC designs.

\section{ACKNOWLEDGMENT}

This work was conducted by Trans.-Wireless Technology Laboratory (TWT Lab.), Hsinchu, Taiwan and ESAT-MICAS, K.U.Leuven, Belgium and sponsored by the National Science Council, Taiwan under the contract: NSC99-2220-E-009-040.

\section{REFERENCES}

[1] S. Bronckers, K. Scheir, G. Van der Plas, G. Vandersteen, Y. Rolain, "A methodology to predict the impact of substrate noise in Analog/RF Systems," IEEE Trans. Computer-Aided Design, vol. 28, pp. 16131626, Nov. 2009.

[2] M. Mendez, D. Mateo, X. Aragones, and J. Gonzalez, "Phase noise degradation of LC-tank VCOs due to substrate noise and package coupling," in Proc. IEEE European Solid-State Circuits Conference ESSCIRC 2005, 12-16 Sept. 2005, pp. 105-108.

[3] H. M. Chao, W. S. Wuen and K. A. Wen, "An Active Guarding Circuit Design for Wideband Substrate Noise Suppression," IEEE Trans. Microw. Theory Tech., vol. 56, no. 11, pp. 2609-2619, Nov. 2008.

[4] T. Tsukada, Y. Hashimoto, K. Sakata, H. Okada, and K. Ishibashi, "An on-chip active decoupling circuit to suppress crosstalk in deepsubmicron CMOS mixed-signal SoCs," IEEE J. Solid-State Circuits, vol. 40 , no. 1 , pp. 67-79, Jan. 2005

[5] W. Winkler, and F. Herzel, "Active Substrate Noise Suppression in Mixed-Signal Circuits Using On-Chip Driven Guard Rings," in Proc. IEEE Custom Integrated Circuits Conf., pp. 357-360, May 2000.

[6] Dai, Haitao and R.W., Knepper, "Modeling and Experimental Measurement of Active Substrate-Noise Suppression in Mixed-Signal 0.18- $\mu \mathrm{m}$ BiCMOS Technology," IEEE Trans. Computer-Aided Design, vol. 28, pp. 826-836, Nov. 2009.

[7] B. de Muer and M. Steyaert, CMOS Fractional-N Synthesizers: Springer, 2003. 